Rev. Int. Contam. Ambie. 35 (Calidad de agua: salud, remediación y perspectiva) 27-36, 2019

DOI: 10.20937/RICA.2019.35.esp03.04

\title{
ANALISIS DE LA BIORREMOCIÓN DE HIDROCARBUROS AROMÁTICOS POLICÍCLICOS EN AGUA POR SPME-GC/MS
}

Analysis of the bioremoval of polycyclic aromatic hydrocarbons in water by SPME-GC/MS

\author{
Esmeralda Guadalupe BLANCO ENRÍQUEZ, Francisco Javier ZAVALA DÍAZ DE LA SERNA, \\ María del Rosario PERALTA PÉREZ, Lourdes BALLINAS CASARRUBIAS y \\ Beatriz Adriana ROCHA GUTIÉRREZ*
}

Facultad de Ciencias Químicas, Universidad Autónoma de Chihuahua, Campus Universitario \#2, Circuito Universitario S/N, Chihuahua, Chihuahua C.P. 31125, México,

*Autora para correspondencia: rochafcq@gmail.com

(Recibido febrero de 2018, aceptado octubre 2018)

Palabras clave: biodegradación, contaminantes orgánicos persistentes, contaminación del agua, microextracción en fase sólida

\section{RESUMEN}

Los contaminantes orgánicos persistentes en el agua como los hidrocarburos aromáticos policíclicos (HAP), representan un riesgo potencial para el ambiente y la salud. La Agencia de Protección Ambiental de los Estados Unidos de Norteamérica (USEPA) clasifica a los HAP pireno, fenantreno y naftaleno dentro de los 16 HAP prioritarios para la USEPA por sus efectos tóxicos, distribución y presencia en el ambiente. El objetivo de este estudio fue evaluar un consorcio microbiano de un desecho de mineral (jales mineros) como una alternativa de eliminación de estos tres HAP en agua. El consorcio se estabilizó utilizando los HAP como única fuente de carbono. La biorremoción se analizó utilizando la técnica ambientalmente amigable de microextracción en fase sólida acoplada a cromatografía de gases y espectrometría de masas (SPME-GC/MS). El porcentaje de recuperación de los HAP se reportó en un rango del 85 al $97 \%$. El porcentaje de eliminación de naftaleno, pireno y fenantreno fue de $99 \%, 98 \%$ y 94 $\%$, respectivamente, a $100 \mathrm{mg} / \mathrm{L}$ después de 14 días de incubación. Los valores de $\mathrm{pH}$ reportados $(\mathrm{pH}=5.2$ - 5.6) pueden indicar la degradación de los HAP en agua. Se concluye que el consorcio microbiano estudiado tiene potencial para la remoción de HAP en agua.

Key words: biodegradation, persistent organic pollutants, solid phase microextraction, water pollution, microextraction

\begin{abstract}
The persistent organic pollutants in water, such as, polycyclic aromatic hydrocarbons (PAHs) represent a potential risk for the environment and human health. The USEPA classified the PAHs pyrene, phenanthrene and naphthalene within the 16 PAH of the priority pollutants for their toxicity, distribution and occurrence in the environment. The aim of this study was to evaluate a microbial consortium from an ore waste as an alternative to remove these three PAHs in water. The consortium was stabilized using
\end{abstract}


the PAHs as the only carbon source. The bioremoval of the PAHs was analyzed using the environmentally friendly technique called solid phase microextraction coupled with gas chromatography and mass spectrometry (SPME-GC/MS). The PAHs percent recoveries were reported in a range of $85-97 \%$. The percentage removal for naphthalene, pyrene and phenanthrene was $99 \%, 98 \%$ and $94 \%$, respectively at a concentration of $100 \mathrm{mg} / \mathrm{L}$ after 14 days of incubation. The $\mathrm{pH}$ values reported $(\mathrm{pH}=5.2-5.6)$ may indicate the PAHs degradation in water. It is concluded that the microbial consortium studied has potential for removal of PAHs from water.

\section{INTRODUCCIÓN}

El agua es, sin duda, uno de los recursos naturales más valiosos del planeta y su calidad está cada vez más deteriorada y escasa debido al incremento de la población, al desarrollo y a la industrialización. Durante los últimos años se ha reportado un incremento de contaminantes orgánicos persistentes en el agua, muchos de los cuales son considerados potencialmente tóxicos para el ambiente y la salud humana, como es el caso de los hidrocarburos aromáticos policíclicos (HAP) (Diaz 2006, Patiño et al. 2014).

Los HAP son compuestos orgánicos que contienen hidrógeno y carbón, los cuales forman anillos aromáticos, suelen ser de color amarillo pálido, blanco o incoloros y se encuentran comúnmente en agua, aire y suelo (Abdel-Shafy y Mansour 2015). Los HAP son considerados contaminantes orgánicos persistentes, hidrofóbicos, altamente estables en el ambiente, se encuentran presentes a bajas concentraciones $(\mu \mathrm{g} / \mathrm{L})$ y su principal riesgo es su capacidad de bioacumulación y biomagnificación.

Los HAP se generan a partir de la combustión incompleta de materiales orgánicos, se liberan directamente al ambiente y se depositan en aire, agua y sedimentos (Haritash y Kaushik 2009). Estos compuestos son peligrosos debido a su presencia, toxicidad, destino y transporte en sistemas acuáticos (Bryer et al. 2006). Con base en los riesgos que representan para la salud, la Agencia de Protección Ambiental de los Estados Unidos de Norteamérica (USEPA) incluyó al pireno, fenantreno y naftaleno, dentro de los 16 HAP como contaminantes de prioridad (Sarria-Villa et al. 2016).

Los HAP pueden estar presentes en el agua por infiltración de descargas de efluentes industriales y domésticas, o por derrames de petróleo que se dan de manera accidental durante su transporte vía marítima (Rengarajan et al. 2015). Los niveles de HAP que se han reportado en aguas superficiales están en concentraciones de $0.05 \mu \mathrm{g} / \mathrm{L}$ a $6,000 \mu \mathrm{g} / \mathrm{L}$. En agua potable las concentraciones se encuentran en un rango de 0.02 a $1.8 \mathrm{ng} / \mathrm{L}$ (Santana et al. 2012). Las concentraciones reportadas en plantas de tratamiento de aguas residuales (PTAR) están en el rango de 13 $170 \mu \mathrm{g} / \mathrm{L}$ a $26380 \mu \mathrm{g} / \mathrm{L}$ (Edokpayi et al. 2016), ya que estos sitios no están diseñados para eliminar este tipo de contaminantes y no se tiene un proceso específico para su remoción (Liu et al. 2011) Vogelsang et al. (2006) estudiaron la eliminación de los 16 HAP prioritarios para la USEPA en una PTAR utilizando sólo el tratamiento químico y reportaron una remocion del 61 al $78 \%$. Estos investigadores observaron que la combinación del tratamiento biológico seguido de un proceso químico de coagulación-floculación resultó en un porcentaje de eliminación del $94 \%$ al $100 \%$. En otro estudio se determinó el porcentaje de biorremoción de los HAP en dos PTAR, la concentración de HAP disminuyó en un 97 a $98 \%$ con el tratamiento biológico (Mezzanotte et al. 2016)Taylor \& Francis Group, LLC.PAH (Polycyclic Aromatic Hydrocarbons. Del mismo modo, se informó una disminución del $78 \%$ de la concentración de HAP en una PTAR después del tratamiento biológico y una eliminación del $99 \%$ en todo el proceso (Burmistrz y Burmistrz 2013). La eficiente eliminación de los HAP en estos estudios, respalda la evidencia de que el tratamiento biológico es eficaz para reducir o eliminar los HAP en el agua.

\section{Procesos de biorremoción para contaminantes orgánicos}

El papel de los microorganismos en la ecología es enorme. El hombre los ha utilizado ampliamente para remediar sitios contaminados (biorremediación) a través de la acumulación, degradación o transformación de desechos peligrosos y productos químicos tóxicos en productos que no afectan al ambiente o a la salud pública (Bautista 2008).

Algunos métodos físicos y químicos aplicados a la degradación de compuestos orgánicos incluyen la destilación con vapor, solidificación, precipitación química, vitrificación e incineración. La principal desventaja de estos métodos es que sólo los trans- 
fieren de un lugar a otro, además de que algunos son costosos y usualmente generan productos más tóxicos que necesitarán un tratamiento secundario (Lizardi et al. 2014). Una alternativa biotecnológica para la remoción de contaminantes orgánicos en el ambiente es representada por el uso de consorcios microbianos. Estos procesos de biodegradación ofrecen la ventaja de ser de bajo o nulo impacto ambiental, debido a la transformación de compuestos aromáticos en productos no peligrosos (ej: $\mathrm{CO}_{2}, \mathrm{H}_{2} \mathrm{O}$ ) y los costos de operación son bajos (Nagarajan y Loh 2014).

Los microorganismos adaptados a los sitios contaminados por actividades relacionadas con el manejo de hidrocarburos de la industria petroquímica, derrames de petróleo, diésel y aceite de motor son candidatos potenciales para la biorremediación. Los microorganismos que se utilizan han pasado por un proceso de selección natural y han adquirido o desarrollado mecanismos especiales que permiten resistir o tolerar altas concentraciones de compuestos orgánicos como los HAP. Esta capacidad para remover los compuestos orgánicos y particularmente los HAP en sitios contaminados ha sido reportada en varios trabajos (Bisht et al. 2015, Ghazali et al. 2004, León y Lizardi 2017, Medina et al. 2014). Sin embargo, en estos estudios los consorcios microbianos han sido aislados de sitios previamente contaminados con estos compuestos, debido a que son microorganismos potencialmente activos. En el caso particular de consorcios provenientes de jales mineros, no se han reportado trabajos en los cuales se realice biorremoción de estos compuestos. Esto podría atribuirse a que comúnmente se utilizan microorganismos ya adaptados a estos contaminantes.

Es importante mencionar que los HAP son susceptibles a procesos de degradación microbiana, generando un gran interés industrial y de investigación en el desarrollo de tecnologías para recuperar agua y suelos contaminados. El objetivo del presente estudio fue evaluar un consorcio microbiano, a partir de jales mineros, los cuales son considerados un desecho minero. Los jales representan una fuente de gran biodiversidad la cual puede ser aprovechada para la biorremoción de pireno, fenantreno y naftaleno en agua.

\section{MATERIALES Y MÉTODOS}

\section{Materiales y reactivos}

En este estudio, se usaron reactivos analíticos y estándares certificados. El naftaleno se adquirió en Sigma-Aldrich (St. Louis, MO, EUA.). El fenantreno y el pireno se adquirieron de Columbia Organic Chemicals Co. (Cassatt SC, EUA), el estándar interno criseno-d12 se compró en AccuStandard (New Haven, CT, EUA). La acetona usada fue grado cromatografía (J.T. Baker). El agua ultrapura (ASTM tipo I) se obtuvo de un sistema de producción FESTA (modelo UP 0999 2017) (Chihuahua, México). Los soportes SPME manuales y las fibras de dimetilsiloxano (PDMS) de $100 \mu \mathrm{m}$, se adquirieron en Suppelco (St. Louis, MO, EUA). Antes del uso, las fibras SPME se acondicionaron según lo recomendado por el fabricante.

\section{Muestreo}

La muestra de jale minero se recolectó en la mina ubicada en Santa Eulalia, Chihuahua, México (latitud $28^{\circ} 60^{\prime} 87^{\prime \prime} \mathrm{N}$ y longitud $\left.105^{\circ} 87^{\prime} 62^{\prime \prime} \mathrm{O}\right)$. Esta zona fue un distrito minero de $\mathrm{Ag}-\mathrm{Pb}-\mathrm{Zn}$ y detuvo las actividades alrededor del año 2010. Las muestras de suelo se recolectaron siguiendo los procedimientos indicados en la NOM-021-RECNAT-2000. La muestra se colectó de una capa de $15 \mathrm{~cm}$ de profundidad en bolsas de polietileno esterilizadas, posteriormente se molieron y se tamizaron a través de un tamiz de tamaño de poro de $0.850 \mathrm{~mm}$ y se almacenaron a $25^{\circ} \mathrm{C}$.

\section{Enriquecimiento del consorcio}

Para seleccionar los microorganismos adaptados a los HAP como única fuente de carbono y energía, se estableció una primera etapa en la cual los jales mineros se incubaron, pesando un gramo de estos y colocándolo en $50 \mathrm{~mL}$ de medio Lebac (2.24 $\mathrm{MgSO}_{4} \cdot 7 \mathrm{H}_{2} \mathrm{O} ; 2.3 \mathrm{~K}_{2} \mathrm{HPO}_{4} ; 5.7 \mathrm{KH}_{2} \mathrm{PO}_{4}$; $7\left(\mathrm{NH}_{4}\right)_{2} \mathrm{SO}_{4}(\mathrm{~g} / \mathrm{L}) ; 1.0$ extracto de levadura y $1 \mathrm{~mL}$ de diésel) en un matraz Erlenmeyer de $250 \mathrm{~mL}$ cubierto con algodón durante dos semanas a temperatura ambiente. Posteriormente el diésel contenido en el medio Lebac fue sustituido por pireno, naftaleno y fenantreno de manera individual como única fuente de carbón y energía, para evaluar la biorremoción en diferentes tiempos (Pérez et al. 2013). La metodología y las condiciones de biorremediación utilizadas en este estudio fueron descritas por Janbandhu y Fulekar (2011 there are many industrial areas discharging effluent containing large amount of polyaromatic hydrocarbon $(\mathrm{PAH})$ remplazando el medio mineral de Tanner por el medio Lebac. El consorcio microbiano seleccionado se expuso a una concentración creciente de fenantreno, naftaleno y pireno comenzando con $5 \mathrm{mg} / \mathrm{L}$ hasta $100 \mathrm{mg} / \mathrm{L}$. Al inicio, se inoculó $1 \mathrm{~mL}$ del consorcio microbiano en matraces Erlenmeyer de $250 \mathrm{~mL}$ que contenían el medio de cultivo Lebac con una concentración del hidrocarburo de interés 
de $5 \mathrm{mg} / \mathrm{L}$. Los matraces inoculados se colocaron en una incubadora de agitación a $120 \mathrm{rpm}$ y temperatura ambiente por 14 días. Después de los 14 días se inoculó un $5 \%(\mathrm{v} / \mathrm{v})$ de suspensión de células bacterianas a otro matraz que contenía medio Lebac con una concentración del hidrocarburo de $10 \mathrm{mg} / \mathrm{L}$. Los matraces se mantuvieron incubados a $120 \mathrm{rpm}$ y temperatura ambiente durante 14 días. El cultivo microbiano se subcultivó en medio Lebac con diferentes concentraciones de los tres hidrocarburos de interés de $20 \mathrm{mg} / \mathrm{L}, 50 \mathrm{mg} / \mathrm{L}$ y $100 \mathrm{mg} / \mathrm{L}$ y se conservó en las mismas condiciones descritas anteriormente en cada concentración. Lo mismo se hizo para los testigos abióticos, los cuales sólo contenían el medio Lebac y el hidrocarburo de interés como se muestra en la figura 1.

\section{Microextracción en fase sólida}

Las técnicas comunes de preparación de muestras, para determinar los HAP en matrices acuosas, incluyen la extracción líquido-líquido (LLE), la extracción ultrasónica (USE) y la extracción en fase sólida (SPE). Sin embargo, estos métodos son costosos, requieren de una gran inversión de tiempo del analista y, debido a todos los pasos que se deben de seguir, se corre el riesgo de perder cantidad del analito en estos procedimientos. Además, no son amigables con el ambiente debido a la gran cantidad de disolventes utilizados.

Una técnica de preparación de muestra libre de solventes es la microextracción en fase sólida (SPME, por sus siglas en inglés), que utiliza fibras de sílice fundida revestidas, montadas en un dispositivo para extraer analitos de matrices sólidas o líquidas. El equilibrio químico se produce en la fibra aplicando calor y agitación de la muestra. En esta etapa, los compuestos afines al material de la fibra son volatizados y adsorbidos en ella. Posteriormente, los analitos se desadsorben térmicamente en el puerto de inyección del cromatógrafo de gases. El modo espacio de cabeza (HS) SPME, se ha aplicado para el análisis de HAP en muestras de agua y suelo. Las ventajas de la SPME incluyen el bajo volumen de muestra solicitada, la eliminación de los pasos previos, la alta recuperación de los analitos y la reutilización de las fibras (Doong et al. 2000a, Doong et al. 2000b, Dias et al. 2013). En este estudio se seleccionó como técnica de extracción a la SPME por sus ventajas y altas recuperaciones de HAP en agua, demostradas en estudios previos (Tuncel y Topal 2011, Corrotea et al. 2014).

Para determinar el tiempo óptimo de extracción se prepararon muestras de agua con una concentración de $50 \mu \mathrm{L} / \mathrm{mL}$. Se evaluaron diferentes tiempos de extracción (30, 45 y $60 \mathrm{~min}$ ) para establecer la mayor recuperación de naftaleno, fenantreno y pireno. Se empleó una fibra de PDMS con recubrimiento de 100 $\mu \mathrm{m}$, con los diferentes tiempos de adsorción y a una temperatura de $60^{\circ} \mathrm{C}$.

\section{Extracción de los HAP por microextracción en fase sólida}

Las muestras de agua se prepararon vertiendo $4900 \mu \mathrm{L}$ del agua con el sobrenadante del experimento de biorremoción el cual contenía fenantreno, pireno o naftaleno y $100 \mu \mathrm{L}$ del estándar interno a una concentración final de $30 \mu \mathrm{g} / \mathrm{L}$ en un vial de 15 $\mathrm{mL}$. Los viales se sellaron con tapas de aluminio y silicona con revestimiento de politetrafluoroetileno (PTFE) y se sumergieron en un baño de arena para mantener una temperatura de $60{ }^{\circ} \mathrm{C}$. Después de 5 min de estabilización a $60{ }^{\circ} \mathrm{C}$, la fibra SPME se expuso a agitación a $1000 \mathrm{rpm}$ en el modo de espacio de cabeza, durante 45 min. Después de la etapa de extracción, la fibra se desadsorbió inmediatamente, en el inyector del cromatógrafo de gases (GC).

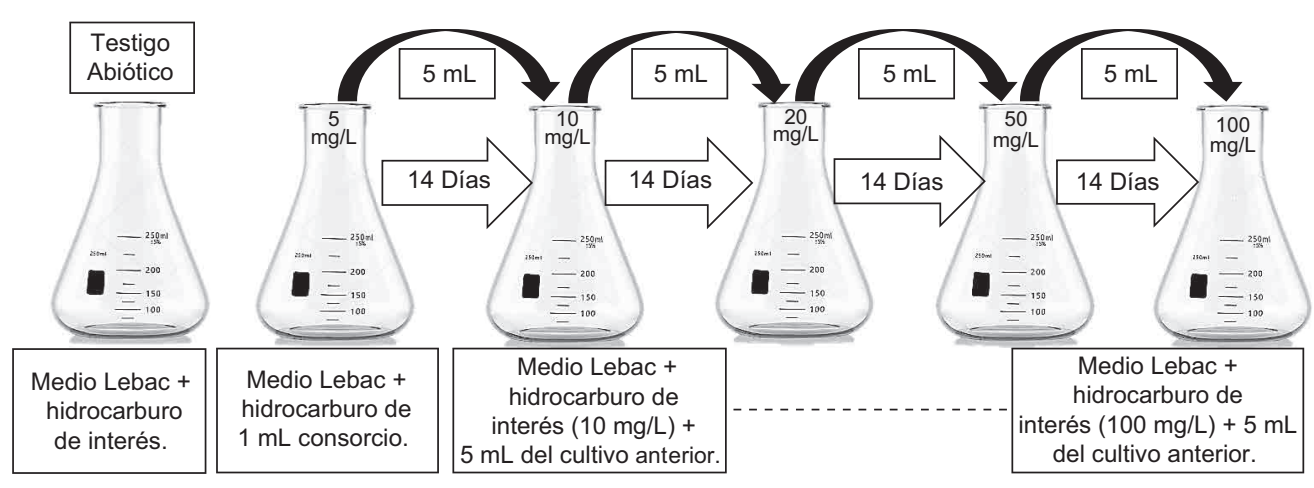

Fig. 1. Experimento de biorremoción para los HAP fenantreno, naftaleno y pireno 


\section{Análisis instrumental}

Los HAP se analizaron con un cromatógrafo de gases (Agilent Techologies7890b, Santa Clara, CA, EUA) acoplado a un espectrómetro de masas (Agilent Technologies 5975C, Santa Clara, CA, EUA). El sistema fue programado en el modo de impacto electrónico $(\mathrm{EI} 70 \mathrm{eV})$. La separación se logró con una columna TG-5HT (30 $\mathrm{mx} 0,25 \mathrm{~mm} \times 0,10 \mu \mathrm{m})$ (Thermo Fisher Scientific, Swedesboro, NJ, EUA) recubrimiento de $5 \%$ de difenil $95 \%$ dimetil polisiloxano. Las muestras se analizaron en el modo división de inyección $(5: 1)$ y utilizando el monitoreo de iones seleccionado (SIM). Se empleó helio de alta pureza $(99.99 \%)$ como gas portador, a una velocidad de $1 \mathrm{~mL} / \mathrm{min}$. La temperatura del horno se programó para $80{ }^{\circ} \mathrm{C}$ durante $1 \mathrm{~min}$ a una velocidad de $15^{\circ} \mathrm{C} / \mathrm{min}$ hasta alcanzar $280^{\circ} \mathrm{C}$, que se mantuvo durante 3 min. La fuente del espectrómetro de masas (MS) y la temperatura cuádruple MS se ajustaron a $230{ }^{\circ} \mathrm{C}$ y $150{ }^{\circ} \mathrm{C}$, respectivamente.

\section{Control de calidad}

El control de calidad de los HAP se evaluó en una curva de calibración de cinco niveles de concentración $(5,10,20,50$ y $100 \mu \mathrm{g} / \mathrm{L})$, incluyendo blancos y triplicados por muestra. El material de vidrio se limpió de acuerdo con el método EPA 610. Se prepararon soluciones patrón estándar a partir de estándares certificados y reactivos grado analítico. El coeficiente de correlación lineal $\left(\mathrm{r}^{2}\right)$, para cada analito fue de 0.98-0.99 para GC/MS. Las desviaciones estándar relativas (RSD) del triplicado por muestra fueron $<20 \%$, lo que indica niveles aceptables de precisión. El porcentaje de recuperación de las muestras de SPME, se calculó agregando el estándar interno (chrsene-d12) a las muestras de agua, para obtener una concentración final de $30 \mu \mathrm{g} / \mathrm{L}$. Las respuestas instrumentales del patrón interno (recuentos de área en el cromatograma de iones total) de las muestras se compararon con la respuesta instrumental del patrón interno a la misma concentración en agua ultrapura. Las tasas de recuperación de los HAP de la extracción de SPME, estuvieron en el rango de 85 a $90 \%$.

\section{RESULTADOS Y DISCUSIÓN}

Tiempo óptimo de extracción de HAP mediante microextracción en fase sólida (SPME)

El tiempo de extracción es una parte importante del desarrollo del método SPME ya que un tiempo adecuado puede mejorar tanto la selectividad como la sensibilidad de la extracción. La figura 2 , muestra los perfiles de extracción obtenidos para los HAP pireno, fenantreno, naftaleno y el estándar interno.

Se observó que para todos los analitos se alcanzó el equilibrio a los 45 min de extracción con espacio de cabeza (HS, por sus siglas en inglés). Las mayores áreas de respuesta se obtuvieron a los 45 min y se reportó que después de este tiempo las áreas de respuesta disminuían. Otros autores han reportado comportamientos similares para el análisis de HAP incluyendo fenantreno, naftaleno y pireno, determinando que el tiempo óptimo para alcanzar el equilibrio de extracción son 45 min (King et al. 2004, Rianawati y Balasubramanian 2009). Otros trabajos sobre el análisis de HAP han empleado eficientemente el modo HS-SPME (Wei y Jen 2007, Dias et al. 2013, Nunes et al. 2012).

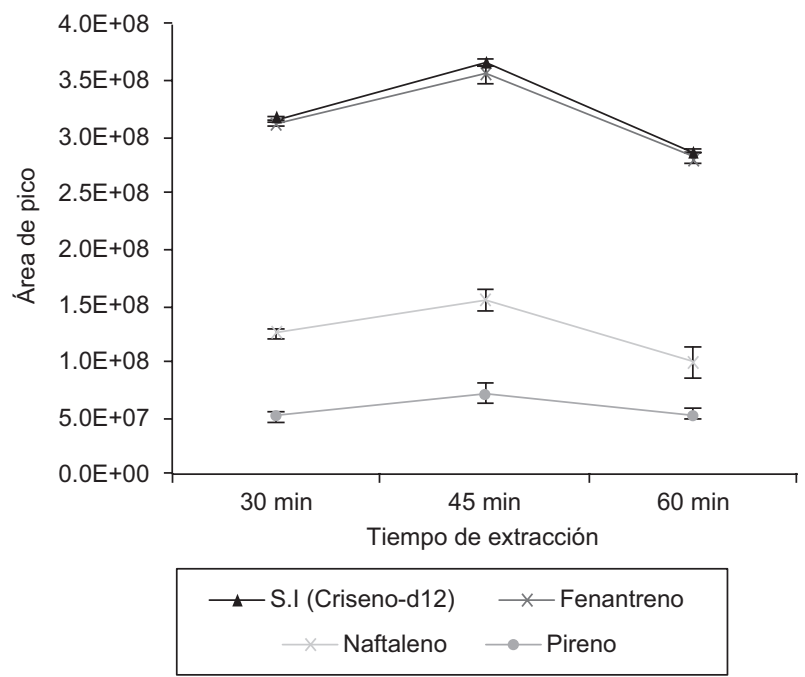

Fig. 2. Comparación del tiempo de extracción con el modo de extracción HS-SPME para todos los HAP y el estándar interno

\section{Porcentajes de recuperación de HAP mediante microextracción en fase sólida (SPME)}

El porcentaje de recuperación para la técnica SPME se calculó para los HAP añadiendo el estándar interno criseno-d12 y comparándolos con testigos abióticos en diferentes concentraciones, los resultados para 5, 10, 20, 50 y $100 \mathrm{mg} / \mathrm{L}$ se muestran en el cuadro $\mathbf{I}$.

Los porcentajes de recuperación para los tres HAP se reportaron dentro de un rango de 85 a $97 \%$. Se observó que el número de anillos en la estructura química de los analitos en estudio no tuvo ningún efecto en la recuperación usando la fibra PDMS de $100 \mu \mathrm{m}$. 
CUADRO I. PORCENTAJE DE RECUPERACIÓN DE LOS HAP NAFTALENO, FENANTRENO Y PIRENO EN EL TIEMPO 0 DE EXPOSICIÓN CON MICOORGANISMOS

\begin{tabular}{cccc}
\hline $\begin{array}{c}\text { Concentración } \\
(\mathrm{mg} / \mathrm{L})\end{array}$ & $\begin{array}{c}\text { Naftaleno } \\
\text { \% recuperación }\end{array}$ & $\begin{array}{c}\text { Fenantreno } \\
\text { \% recuperación }\end{array}$ & $\begin{array}{c}\text { Pireno } \\
\text { \% recuperación }\end{array}$ \\
\hline 5 & 89 & 88 & 91 \\
10 & 96 & 97 & 97 \\
20 & 85 & 87 & 87 \\
50 & 90 & 91 & 90 \\
100 & 97 & 95 & 88 \\
\hline
\end{tabular}

Esto podría deberse a que la SPME es una técnica que proporciona en un sólo paso etapas múltiples de procedimientos (extracción, selección y preconcentración) por lo que disminuye la pérdida de analitos, como ocurre generalmente en métodos de extracción tradicionales como es el caso de la extracción líquidolíquido (Rianawati y Balasubramanian 2009).

\section{Remoción de HAP mediante el uso de microorga- nismos provenientes de jales mineros}

Estudios previos de biorremoción utilizaron consorcios microbianos aislados provenientes de sitios contaminados por HAP relacionados con la industria petroquímica, derrames de petróleo, diésel y aceite de motor. Por ejemplo, los investigadores Janbandhu y Fulekar (2011 there are many industrial areas discharging effluent containing large amount of polyaromatic hydrocarbon (PAH) reportaron una degradación total $(100 \%)$ de fenantreno en agua en 14 días de incubación utilizando un consorcio recolectado de un suelo de industrias petroquímicas y refinerías de petróleo. Jacques et al. (2008) mostraron una remoción para fenantreno del $99 \%$ a $500 \mathrm{mg} / \mathrm{L}$ y del $96 \%$ a una concentración de 1000 $\mathrm{mg} / \mathrm{L}$, después de 70 días de incubación, usando un consorcio de una industria petroquímica. Tirado et al. (2015) reportaron una remoción del $56 \%$ de pireno en un sitio contaminado con $100 \mathrm{mg} / \mathrm{L}$ en 28 días, utilizando un consorcio aislado de composta.

En el presente estudio, los microorganismos se adaptaron a partir de jales mineros para usar fenantreno, pireno y naftaleno como la única fuente de carbono. En comparación con los estudios citados los microorganismos ya estaban adaptados a los hidrocarburos, ya que se aislaron directamente de sitios contaminados con estos compuestos, obteniendo resultados similares. Las mayores remociones se informaron después de 14 días de actividad microbiana, eliminando hasta $97 \%$ para los tres HAP después de 56 días de adaptación para el consorcio microbiano. Este resultado es similar al obtenido por Janbandhu quien también probó 14 días de incubación obteniendo $100 \%$ de remoción. El porcentaje de remoción de fenantreno, naftaleno y pireno a $5 \mathrm{mg} / \mathrm{L}$ fue del $62 \%$, $77 \%$ y $78 \%$ respectivamente. La mayor remoción se reportó a $100 \mathrm{mg} / \mathrm{L}$ con porcentajes de $97 \%$ a $99 \%$ en 14 días de incubación como se muestra en la figura 3. Este resultado fue mayor al reportado por Tirado et al. (2015) quienes lograron únicamente una remoción del $56 \%$ en el doble de tiempo a la misma concentración.

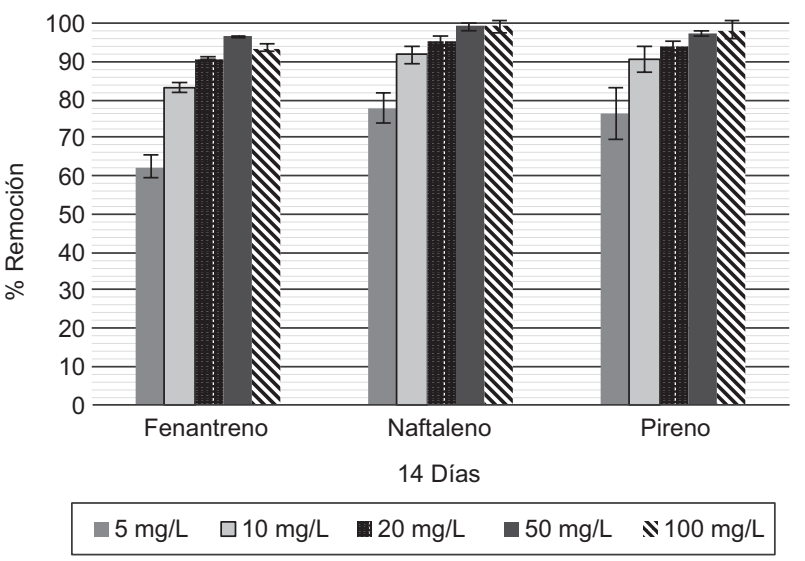

Fig. 3. Porcentaje de remoción de fenantreno, naftaleno y pireno después de 14 días de exposición con los microorganismos a diferentes concentraciones. Las barras de error son desviación estándar relativa (RSD) $<20 \%$

El alto porcentaje de remociones de HAP logrado por el consorcio proveniente de los jales mineros, presentado en este estudio es comparable a los estudios citados, lo que demuestra que la biorremediación es una alternativa para eliminar los HAP de bajo peso molecular en el agua.

Los menores porcentajes de remoción se reportaron en la concentración más baja $(5 \mathrm{mg} / \mathrm{L})$. Este resultado pudiera atribuirse a la etapa de adaptación inicial de los consorcios microbianos ya que se observó que el porcentaje de remoción aumentó al tener una 
CUADRO II. ESTRUCTURA QUÍMICA Y PROPIEDADES DE LOS HAP ANALIZADOS

\begin{tabular}{lcccc}
\hline Compuesto & Fórmula & Kow & \% de Biorremoción \\
\hline Naftaleno & $\mathrm{C}_{10} \mathrm{H}_{8}$ & 3.29 & 99 \\
Fenantreno & $\mathrm{C}_{14} \mathrm{H}_{10}$ & 4.46 & 94 \\
Pireno & $\mathrm{C}_{16} \mathrm{H}_{10}$ & 4.88 & 98 \\
\hline
\end{tabular}

mayor concentración de HAP, debido a que el consorcio se logró adaptar a los HAP como única fuente de carbono. Las remociones más altas se reportaron para el naftaleno ( $99 \%$ ), esto podría deberse a que de los tres HAP analizados, éste presenta la estructura química menos compleja, que consiste en dos anillos de benceno, con un coeficiente de partición de agua octanol $\left(\mathrm{K}_{\mathrm{ow}}\right)$ de 3.29. Mientras que el fenantreno y el pireno tienen propiedades químicas muy similares (tres anillos de benceno, $\mathrm{K}_{\mathrm{ow}}=4.46 \mathrm{y}$ cuatro anillos de benceno, $\mathrm{K}_{\mathrm{ow}}=4.88$, respectivamente) como se muestra en el cuadro II.

El coeficiente de partición octanol-agua $\left(\mathrm{K}_{\mathrm{ow}}\right)$ indica la solubilidad de los compuestos orgánicos en el agua y su disposición en el ambiente, cuando $\mathrm{K}_{\mathrm{ow}}$ $>1$ los compuestos son hidrofóbicos, mientras que un $\mathrm{K}_{\mathrm{ow}}<1$ indica que los compuestos son hidrofílicos. Se ha reportado que los HAP con $\mathrm{K}_{\text {ow }}$ mayor a seis son considerados altamente hidrofóbicos $(\mathrm{Li}$ et al. 2008). En los HAP analizados en el presente estudio el $\mathrm{K}_{\mathrm{ow}}$ fue menor a seis lo que podría indicar que se trata de compuestos más solubles en agua y su tendencia en acumularse en los sedimentos es elevada.

\section{Efecto del pH}

Los cambios de $\mathrm{pH}$ en los medios de cultivo pueden indicar actividad metabólica como resultado de la producción de metabolitos ácidos a partir de la degradación de los HAP (Janbandhu y Fulekar 2011)there are many industrial areas discharging effluent containing large amount of polyaromatic hydrocarbon (PAH. El crecimiento del consorcio durante los 14 días de exposición a los HAP causó una disminución en el $\mathrm{pH}$ de 7 a 5.2, 5.4 y 5.6 para naftaleno, fenantreno y pireno, respectivamente a 100 $\mathrm{mg} / \mathrm{L}$, mientras que el testigo abiótico no presentó un cambio en pH, permaneciendo en 7 (Fig. 4). Dado que el naftaleno presentó el mayor porcentaje de eliminación, podría ser posible que este HAP produjera la mayor cantidad de metabolitos ácidos.

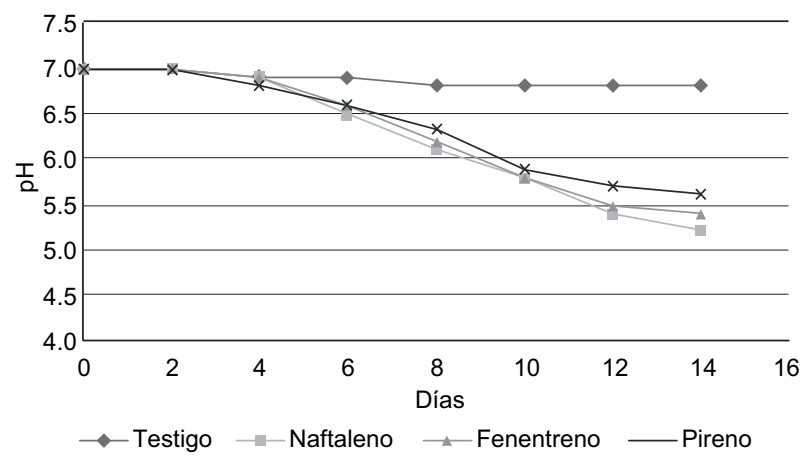

Fig. 4. Cambios en el pH durante el proceso de remoción de HAP mediante un consorcio microbiano

Según Olajire y Essien (2014) la degradación más rápida y completa de los contaminantes orgánicos, como los hidrocarburos, se logra en condiciones aeróbicas. Los contaminantes orgánicos actuarán como productos intermedios en el metabolismo del ácido tricarboxílico para generar energía que se utilizará para los microorganismos. En este experimento, después del día 12 de incubación, sólo se observó una ligera disminución en el $\mathrm{pH}$ para el naftaleno. El mismo tiempo de incubación fue reportado por Janbandhu y Fulekar (2011 there are many industrial areas discharging effluent containing large amount of polyaromatic hydrocarbon $(\mathrm{PAH})$ para eliminar la cantidad total de fenantreno presente en el agua analizada. 
Kim et al. (2005) reportaron que la degradación de HAP por Mycobacterium vanbaalenii ocurre en un $\mathrm{pH}$ ácido. Yuan et al. (2000) informaron que un $\mathrm{pH}$ entre 6 y 7 es necesario para iniciar la degradación de fenantreno, pireno y acenafteno. $\mathrm{El} \mathrm{pH}$ ácido reportado en este estudio (5.2 - 5.6), puede indicar la mayor degradación de los HAP analizados a los 14 días utilizando el consorcio de jales mineros.

Los microorganimos reportados en la bibliografía que han degradado a los HAP a este $\mathrm{pH}$ son Pseudomonas aeruginosa, Pseudomons fluoresens, Mycobacterium spp., Haemophilus spp., Rhodococcus spp., Paenibacillus spp. (Haritash y Kaushik 2009), por lo cual se cree que estos microorganismos pudieran estar presentes en la muestra de jal minero.

\section{CONCLUSIONES}

El uso de las técnicas de biorremediación, en este caso con jales mineros para disminuir o eliminar del agua los HAP naftaleno, pireno y fenantreno, fue altamente eficiente. Se reportó un porcentaje de remoción de hasta el $90 \%$ a una concentración de $100 \mathrm{mg} / \mathrm{L}$ en agua y 14 días de incubación de los HAP. El porcentaje de recuperación para los tres HAP analizados, utilizando la técnica de extracción de SPME, fue eficiente al obtener porcentajes en un rango del $84 \%$ al $97 \%$ de recuperación. Por otro lado, la disminución en el pH indicó que el consorcio de los jales mineros presentó actividad metabólica debido a la producción de metabolitos ácidos, lo que indica una degradación de los HAP. Se recomienda realizar estudios específicos para la identificación de los microorganismos responsables de la biodegradación. Para los autores, este es el primer estudio que utiliza un consorcio a partir de jales mineros y es analizado por la técnica SPME-GC/MS.

\section{REFERENCIAS}

Abdel-Shafy H.I. y Mansour S.M. (2015). A review on polycyclic aromatic hydrocarbons: Source, environmental impact, effect on human health and remediation. Egyptian Journal of Petroleum. 2-17.

DOI: 10.1016/j.ejpe.2015.03.011

Bautista-Hernández D.A. (2008). Bioadsorción de Metales Pesados Mediante el Uso de Biomasa Bacteriana Aislada de Jales Mineros-Edición Única. Tesis de Maestria. Instituto Tecnológico y de Estudios Superiores de Monterrey. Estado de México, 107 pp. [en línea] http://hdl.handle.net/11285/569087 10/11/17.
Bisht S., Pandey P., Bhargava B., Sharma S., Kumar V. y Krishan D. (2015). Bioremediation of polyaromatic hydrocarbons (PAHs) using rhizosphere technology. Brazilian Journal of Microbiology 46 (1), 7-21. DOI: 10.1590/S1517-838246120131354

Bryer P. J., Elliott, J. N., y Willingham, E. J. (2006). The effects of coal tar based pavement sealer on amphibian development and metamorphosis. Ecotoxicology 15 (3), 241-247. DOI: 10.1007/s10646-005-0055-z

Burmistrz P. y Burmistrz M. (2013). Distribution of polycyclic aromatic hydrocarbons in coke plant wastewater. Water Science and Technology 68 (11), 2414-2420. DOI: 10.2166/wst.2013.506

Corrotea Y., Sánchez K., Rubio M. A. y Richter P. (2014). Extraction of Polycyclic Aromatic Hydrocarbons From Water Samples Into a Rotating-Disk Microextractor and the Subsequent Determination By Gas. chemical soc. 2, 2477-2480.

Dias A. N., Sim V., Merib, J., y Carasek, E. (2013). Cork as a new (green) coating for solid-phase microextraction: Determination of polycyclic aromatic hydrocarbons in water samples by gas chromatography-mass spectrometry. Analytica Chimica Acta 772, 33-39.

DOI: 10.1016/j.aca.2013.02.021

Diaz N.E. (2006). Desarrollo, Validacion Y Aplicacion De Metodos Cromatograficos Para La Determinacion De Hidrocarburos Aromaticos Policiclicos En Fuentes De Abastecimiento De Agua Potable De La Ciudad De Monterrey. [en línea] http://eprints.uanl. $\mathrm{mx} / 5873 / 1 / 1080128444 . P D F .15 / 10 / 17$.

Doong R., Chang S., y Sun Y. (2000a). Solid-phase microextraction and headspace solid-phase microextraction for the determination of high molecular-weight polycyclic aromatic hydrocarbons in water and soil samples. Journal of chromatographic science 38(12), 528-534.

Doong R., Chang S., y Sun Y. (2000b) Solid-phase microextraction for determining the distribution of sixteen US Environmental Protection Agency polycyclic aromatic hydrocarbons in water samples, 879, 177-188.

Edokpayi J. N., Odiyo J.O., Popoola O.E., y Msagati A.M. (2016). Determination and Distribution of Polycyclic Aromatic Hydrocarbons in Rivers, Sediments and Wastewater Effluents in Vhembe District, South Africa. International Journal of Environmental Research and Public Health 13. DOI: 10.3390/ijerph13040387

Ghazali F.M., Abdul-Rahman R.N.Z., Salleh A.B., y Basri M. (2004). Biodegradation of hydrocarbons in soil by microbial consortium. International Biodeterioration and Biodegradation 54(1), 61-67.

DOI: $10.1016 /$ j.ibiod.2004.02.002

Haritash A.K. y Kaushik C.P. (2009). Biodegradation aspects of Polycyclic Aromatic Hydrocarbons (PAHs ): A review. Journal of Hazardous Materials, 
169, 1-15. [en linea] https://sci-hub.cc/10.1016/j. jhazmat.2009.03.137.

DOI: 10.1016/j.jhazmat.2009.03.137

Jacques J.S., Okeke B.C., Bento F.M., Teixeira A.S., Peralba C.R. y Camargo A.O. (2008). Microbial consortium bioaugmentation of a polycyclic aromatic hydrocarbons contaminated soil. Bioresource Technology 99 (7), 2637-2643. DOI: 10.1016/j.biortech.2007.04.047

Janbandhu A. y Fulekar M.H. (2011). Biodegradation of phenanthrene using adapted microbial consortium isolated from petrochemical contaminated environment. Journal of Hazardous Materials 187(1-3), 333-340. DOI: 10.1016/j.jhazmat.2011.01.034

Kim Y.H., Freeman J.P., Moody J.D., Engesser K.H. y Cerniglia C.E. (2005). Effects of $\mathrm{pH}$ on the degradation of phenanthrene and pyrene by Mycobacterium vanbaalenii PYR-1. Applied Microbiology and Biotechnology 67(2), 275-285. DOI: $10.1007 / \mathrm{s} 00253-004-1796-y$

King A J., Readman J.W. y Zhou J.L. (2004). Determination of polycyclic aromatic hydrocarbons in water by solid-phase microextraction - gas chromatography mass spectrometry $\mathbf{5 2 3}, 259-267$.

DOI: 10.1007/s00253-004-1796-y

León-Borges J.A. y Lizardi-Jimenéz M.A. (2017). Hydrocarbon pollution in underwater sinkholes of the Mexican Caribbean caused by tourism and asphalt : Historical data series and cluster analysis Jose. Tourism Management 63, 179-186.

DOI: 10.1016/j.tourman.2017.06.018

Li L., Xie S., Cai H., Bai X., y Xue Z. (2008). Quantitative structure-property relationships for octanol-water partition coefficients of polybrominated diphenyl ethers. Chemosphere 72(10), 1602-1606.

DOI: $10.1016 /$ j.chemosphere.2008.04.020

Liu J.J., Wang X.C. y Fan B. (2011). Characteristics of PAHs adsorption on inorganic particles and activated sludge in domestic wastewater treatment. Bioresource Technology 102(9), 5305-5311.

DOI: 10.1016/j.biortech.2010.12.063

Lizardi-Jiménez M.A., Leal-Bautista R.M., Ordaz A. y Reyna-Velarde R. (2014). Airlift bioreactors for hydrocarbon water pollution remediation in a tourism development pole. Desalination and Water Treatment 1-6. DOI: 10.1080/19443994.2013.876670

Medina-Moreno S.A., Jiménez-González A., GutiérrezRojas M. y Lizardi-Jiménez M.A. (2014). Hydrocarbon Pollution Studies of Underwater Sinkholes Along Quintana Roo As a Function of Tourism Development in the Mexican Caribbean. Revista Mexicana de Ingeniería Química 13, 509-516.

Mezzanotte V., Anzano M., Collina E., Marazzi F.A. y Lasagni M. (2016). Distribution and Removal of
Polycyclic Aromatic Hydrocarbons in Two Italian Municipal Wastewater Treatment Plants in 2011-2013. Polycyclic Aromatic Compounds 36(3).

DOI: $10.1080 / 10406638.2014 .957409$

Nagarajan K. y Loh K.C. (2014). Formulation of microbial cocktails for BTEX biodegradation. Biodegradation. 26(1), 51-63. DOI: 10.1007/s10532-014-9715-0

NOM-021-RECNAT-2000 (2000) Segunda Seccion. Diario Oficial de la Federación. [en línea] http://www. ordenjuridico.gob.mx/Documentos/Federal/wo69255. pdf. 20/10/17.

Nunes J., Nardini G., Merib J., Neves A., Martendal E. y Carasek E. (2012). Simultaneous determination of polycyclic aromatic hydrocarbons and benzene, toluene, ethylbenzene and xylene in water samples using a new sampling strategy combining different extraction modes and temperatures in a single extraction solidphase microextraction-gas chromatography - mass spectrometry procedure. Journal of Chromatography A 1233, 22-29.

DOI: $10.1016 /$ j.chroma.2012.02.022

Olajire A.A. y Essien J.P. (2014). Aerobic Degradation of Petroleum Components by Microbial Consortia. Journal of Petroleum \& Environmental Biotechnology 5(5), 1-22. DOI: 10.4172/2157-7463.1000195

Patiño Y., Díaz E. y Ordóñez S. (2014). Water Micropollutants: Classification And Treatment Technologies. Avances en Ciencias e Ingeniería 5, 1-20. [en línea] http://www.redalyc.org/articulo.oa?id=323631115001. 10/10/17

Pérez-Armendriz B., Mauricio-Gutirrez A., Jimnez-Salgado T., Tapia-Hernndez A. y Santiesteban-Lopez A. (2013). Emulsification of Hydrocarbons Using Biosurfactant Producing Strains Isolated from Contaminated Soil in Puebla, Mexico. Biodegradation Engineering and Technology 25-45. DOI: 10.5772/56143

Rengarajan T., Rajendran P., Nandakumar N., Lokeshkumar B., Rajendran P. y Nishigaki I. (2015). Asian Pacific Journal of Tropical Biomedicine. Asian Pacific Journal of Tropical Biomedicine 5(3), 182-189.

DOI: 10.1016/S2221-1691(15)30003-4

Rianawati E. y Balasubramanian R. (2009). Optimization and validation of solid phase micro-extraction (SPME) method for analysis of polycyclic aromatic hydrocarbons in rainwater and stormwater. Physics and Chemistry of the Earth 34(13-16), 857-865.

DOI: $10.1016 /$ j.pce.2009.07.003

Santana-Romero J.L., Valdés-Callado M. y Lima O.L. (2012). Determinación de hidrocarburos aromáticos policíclicos ligeros en aguas superficiales de los ríos Almendares y Luyanó en La Habana. Revista CENIC Ciencias Químicas 43, 1-7. [en línea] http://www. redalyc.org/articulo.oa?id=181628775002 10/10/17. 
Sarria-Villa R., Ocampo-Duque W., Páez M. y Schuhmacher M. (2016). Science of the Total Environment Presence of PAHs in water and sediments of the Colombian Cauca River during heavy rain episodes, and implications for risk assessment. Science of the Total Environment 540, 455-465. DOI: $10.1016 /$ j.scitotenv.2015.07.020

Tirado-Torres D., Acevedo-Sandoval O., Romo-Gómez C. y Marmolejo-Santillán Y. (2015). Participación de consorcios microbianos en la biodegradación de hidrocarburos aromáticos policíclicos. Revista Iberoamericana de Ciencias. 2, 77-86. [en línea] https://www.researchgate. net/publication/296698154_Participacion_de_Consorcios_Microbianos_en_la_Biodegradacion_de_Hidrocarburos_Aromaticos_Policiclicos 15/10/17.

Tuncel S.G. y Topal T. (2011). Multifactorial Optimization Approach for Determination of Polycyclic Aromatic Hydrocarbons in Sea Sediments of Turkish Mediterranean Coast. American Journal of Analytical Chemistry 2(7), 783-794. DOI: 10.4236/ajac.2011.27090
Vogelsang C., Grung M., Jantsch T.G., Tollefsen K.E. y Liltved H. (2006). Occurrence and removal of selected organic micropollutants at mechanical, chemical and advanced wastewater treatment plants in Norway Water Research. 40(19), 3559-3570. DOI: $10.1016 /$ j.watres.2006.07.022

Wei M. y Jen J. (2007). Determination of polycyclic aromatic hydrocarbons in aqueous samples by microwave assisted headspace solid-phase microextraction and gas chromatography / flame ionization detection. Talanta 72, 1269-1274. DOI: 10.1016/j.talanta.2007.01.017 Yuan S.Y., Wei S.H. y Chang B.V. (2000). Biodegradation of polycyclic aromatic hydrocarbons by a mixed culture. Chemosphere 41(9), 1463-1468.

DOI: $10.1016 / \mathrm{S} 0045-6535(99) 00522-6$ 